\title{
Herdabilidades de Parâmetros de Curvas de Crescimento Não-Lineares em Zebuínos, no Estado de Pernambuco ${ }^{1}$
}

\author{
Kleber Régis Santoro², Severino Benone Paes Barbosa ${ }^{3}$, Eufrázio de Souza Santos4 ${ }^{4}$ Lúcia \\ Helena de Albuquerque Brasil $^{5}$
}

\begin{abstract}
RESUMO - Objetivou-se estimar parâmetros genéticos e fenotípicos de curvas de crescimento de modelos não-lineares. Foram analisados dados de pesagem constantes no banco de dados de Controle de Desenvolvimento Ponderal da Associação Brasileira de Criadores de Zebu (ABCZ), referentes a 24.028 animais Zebu, nascidos entre 1960 e 2000, das raças Guzerá, Nelore e Nelore Mocho. As pesagens ocorreram ao nascimento e em intervalos de 90 dias até dois anos de idade. Os seguintes modelos não-lineares foram utilizados na análise dos dados de peso-idade: Brody, Gompertz, Logístico, von Bertalanffy e Richards. Os efeitos fixos estudados no modelo misto foram sexo, rebanho, ano e mês de nascimento e regime de criação. As herdabilidades para os parâmetros foram de baixa a alta magnitude, em geral, para todos os modelos. As correlações genéticas entre peso assintótico e taxa de maturidade e entre peso assintótico e velocidade de crescimento foram negativas, enquanto aquelas entre taxa de maturidade e velocidade de crescimento foram positivas. As correlações fenotípicas foram negativas entre peso assintótico e taxa de crescimento e entre peso assintótico e velocidade de crescimento e positivas entre taxa e velocidade de crescimento. Encontrou-se variabilidade possível de ser explorada em um programa de melhoramento genético, especialmente para a raça Nelore, que apresentou amostra de dados e resultados mais consistentes.
\end{abstract}

Palavras-chave: componentes de variância, Guzerá, maturidade, Nelore, Nelore Mocho, zebu

\section{Heritabilities of Nonlinear Growth Curve Parameters in Zebu Breeds, in Pernambuco State, Northeastern Brazil}

\begin{abstract}
Weight records of 24.028 zebu animals from Guzerá, Nelore, and Polled Nelore breeds available from Brazilian Association of Zebu Breeders (ABCZ) database were used to estimate heritabilities of growth curve parameters. Non-linear Brody, Gompertz, Logistic, Mitscherlich, von Bertalanffy, Richards, and Double Logistic models including sex, farm, year of birth, month of birth, raising system, and interaction sex*raising system as fixed effects and sire and dam, as random effects were adjusted using weightage records of animals which were born between 1960 and 2000, every three months, from birth to 24 months of age. Heritability estimates of growth curve parameters ranged from low to high values for all models and breeds. Genetic correlations between asymptotic weight and mature rate and asymptotic weight and growth rate were negative whereas those between mature rate and growth rate were positive. Phenotypic correlations between asymptotic weight and mature rate and asymptotic weight and growth rate were negative and those between mature rate and growth rate were positive. The variability observed in this study indicates the possibility of changing the growth curve by a breeding program, especially for the Nellore because more consistent records and results obtained for this breed.
\end{abstract}

Key Words: Guzerá, Nelore, Nelore Mocho, maturity, variance components, zebu

\section{Introdução}

O estudo das funções matemáticas não-lineares da família de Richards utilizadas para descrever o crescimento corporal animal, especialmente em bovinos, é de uso amplo e corrente na literatura nacional (Ludwig et al., 1981; Nobre et al., 1987; Kroll \& Tornero, 1994; Perotto et al., 1994; Freitas et al., 1997; Perotto et al., 1997; Oliveira et al., 2000; Mazzini, 2001; Lôbo \& Martins Filho, 2002) e estran- geira (Brown et al., 1976; Fitzhugh Jr., 1976; Goonewardene et al., 1981; DeNise \& Brinks, 1985; Koenen \& Groen, 1996; Kaps et al., 2000).

O benefício do uso de modelos não-lineares no melhoramento genético animal poderia ser observado na estimação dos parâmetros para as curvas, proporcionando a identificação dos animais seriam mais adequados para determinados fins de seleção, como maior taxa de crescimento em determinada fase da vida.

\footnotetext{
${ }^{1}$ Parte da tese de doutorado do primeiro autor financiada pelo CNPq.

2 Professor - UAG/UFRPE. Rua Ernesto Dourado, 82 - Bairro Heliópolis - CEP: 55296-190 - Garanhuns - PE. E.mail: ksantoro@ufrpe.br

3 Professor DZ/UFRPE. Rua Dom Manoel de Medeiros, s/n; Bairro de Dois Irmãos; 52171-900, Recife (PE). E.mail: sbarbosa@ufrpe.br

4 Professor Pós-Graduação em Biometria/UFRPE. Rua Dom Manoel de Medeiros, s/n; Bairro de Dois Irmãos; 52171-900, Recife (PE).

5 Professora DZ/UFRPE. Rua Dom Manoel de Medeiros, s/n; Bairro de Dois Irmãos; 52171-900, Recife (PE).
} 
Além dos parâmetros que poderiam ser analisados diretamente da equação ( $a, k$ e $m$ ) e que apresentam interpretação biológica, poderiam ser deduzidos como auxílio ao melhoramento genético para a equação de Richards (Perotto et al., 1997) os índices taxa média de crescimento absoluto, taxa média de maturação absoluta, peso, grau de maturidade e idade no ponto de inflexão, máxima velocidade de ganho de peso e máxima taxa de maturação.

Oscomponentes genéticos das populações e os parâmetros ambientais que agem sobre o desempenho dos animais (Owens et al., 1993) são fundamentais no cálculo do valor genético dos animais, na seleção e na condução de programas de melhoramento.

Entretanto, são poucos os trabalhos sobre herdabilidade e outros componentes genéticos relacionados aos parâmetros biologicamente interpretáveis dessas equações, uma vez que a maioria dos autores estudou somente o ajuste das curvas, disponibilizando apenas os valores médios dos parâmetros para as amostras analisadas (DeNise \& Brinks, 1985; Koenen \& Groen, 1996; Lôbo \& Martins Filho, 2002).

A escassez de informações dificulta o trabalho de seleção dentro e entre rebanhos, não só em razão das diferenças de condições ambientais e de criação a que estariam submetidos os animais, mas também pela diferença entre os genótipos.

Este trabalho foi realizado para se estimar os componentes de variância genética relacionados aos parâmetros das curvas de crescimento de animais zebuínos das raças Guzerá, Nelore e Nelore Mocho criados no estado de Pernambuco.

\section{Material e Métodos}

Entre 24.028 animais Zebu das raças Guzerá (4.436), Nelore (14.035) e Nelore Mocho (5.557), nascidos entre 1960 e 2000 e criados no estado de Pernambuco, selecionaram-se aqueles que possuíam nove pesagens (realizadas em intervalos de, aproximadamente, 90 dias) do nascimento aos dois anos de idade. Portanto, a amostra inicial foi reduzida para 1.358 animais (198 Guzerá, 928 Nelore e 232 Nelore Mocho), contabilizando-se um total de 12.222 observações de peso-idade. As informações constavam no banco de dados de Controle de Desenvolvimento Ponderal da Associação Brasileira de Criadores de Zebu (ABCZ).
Os animais foram criados em sistemas de criação: a pasto, semi-estabulado, estabulado e com mudança de um sistema para outro.

As funções pertencentes à família de Richards foram agrupadas por Richards (1959), que agregou algumas funções de crescimento já conhecidas e utilizadas na análise de dados individuais de animais, plantas e populações em uma única função flexível pela qual poderiam ser deduzidas. Entre os casos especiais da família de Richards, encontram-se as funções de Brody ou Monomolecular (Brody, 1945), Logística ou Autocatalítica (Nelder 1961), von Bertalanffy (von Bertalanffy, 1957) e Gompertz (Winsor, 1932), com os parâmetros $a, b$ e $k$, e a de Richards (Richards, 1959), com um parâmetro a mais, o $m$.

A função geral é dada por:

$$
Y_{t}=a\left(1 \pm b e^{-k t}\right)^{m}
$$

em que, segundo Fitzhugh Jr. (1976) e Nobre et al. (1987), de forma generalizada, considera-se que $Y_{t}$ é o peso na idade $t$; $a$, o valor assintótico, interpretado como peso assintótico ou peso à maturidade; $b$, um parâmetro escala (constante de integração), sem interpretação biológica; $k$, o índice de maturidade ou de precocidade; $k^{-1}$, o tempo para atingir a maturidade; $m$, o parâmetro de inflexão, que estabelece o grau de maturidade $u_{I}$, no ponto de inflexão $u_{I}=[(m-1) / m]^{m}$, sendo $u_{t}$ a proporção peso/idade $t: u_{t}=Y_{t} / a=\left(1 \pm b e^{-k t}\right)^{m}$. O sinal inferior de \pm deve ser aplicado quando $m \geq 1$, e o superior, quando $m<0$.

O parâmetro $m$ assumiria os seguintes valores para os casos especiais da família de Richards: Brody (1), von Bertalanffy (3), Gompertz $(\mu \rightarrow \infty)$ e Logístico $(-1)$. Assim, foram utilizados os seguintes modelos não-lineares para análise dos dados de peso-idade:

Brody (B): $P_{i}=a-b e^{-k t_{i}}$

Gompertz (G): $P_{i}=a e^{-b e^{-k t_{i}}}$

Logístico (L): $P_{i}=a\left(1+b e^{-k t_{i}}\right)^{-1}$

von Bertalanffy (vB): $P_{i}=a\left(1-b e^{-k t_{i}}\right)^{3}$

Richards (R): $P_{i}=a\left(1-b e^{-k t_{i}}\right)^{m}$

em que $P_{i}$ representa o peso observado no tempo $t_{i}$; $t_{i}$, o tempo decorrido do nascimento até a pesagem, em dias; $a$, o peso assintótico; $b$, uma constante de integração; $k$, o índice de maturidade e $m$, o parâmetro de inflexão.

Empregou-se nas análises o PROC NLIN, do software SAS (SAS, 2000), para o ajuste dos modelos não-lineares. As estimativas iniciais foram obtidas na 
literatura consultada ou pela média dos dados da amostra para todos os parâmetros, quando não disponíveis na literatura. Optou-se pelo método de estimação de Gauss-Newton para o cálculo das estimativas dos parâmetros, pois seria mais simples que o de Newton, mesmo necessitando-se de maior número de iterações (Hoffman \& Vieira, 1998). O coeficiente de ajuste $\left(r^{2}\right)$ para as equações foi calculado como $r^{2}=1-($ sqe)/ (sqt), em que sqe é a soma de quadrados dos erros e sqt, a soma de quadrados total (Kvålseth, 1985).

As estimativas obtidas para os parâmetros das equações que convergiram e se encontravam dentro de limites considerados biologicamente aceitáveis, ou seja, $a \leq 1000$ e $k \leq 1$, foram utilizadas para o cálculo da herdabilidade e das correlações.

As estimativas de herdabilidades para os parâmetros das equações foram obtidas por máxima verossimilhança restrita, pelo programa DFREML 3.1 (Meyer, 2000), utilizando-se o seguinte modelo misto multivariado:

$$
y=\underset{\sim}{\beta}+\underset{\sim}{\alpha \underset{\sim}{\alpha}} \underset{\sim}{\varepsilon}
$$

em que: $y$ é um vetor de variáveis dependentes, sendo $a, b$ e $k$ para os modelos Brody, Gompertz, Logístico e Von Bertalanffy e $a, b, k$ e $m$ para o modelo Richards; $X$, uma matriz de incidência de efeitos fixos definida por zeros e uns, considerando-se o sexo ( 1 = macho, 2 = fêmea), a propriedade $(7, \ldots, 41)$, o ano $(1970, \ldots, 1999)$ e o mês de nascimento $(1, \ldots, 12)$ e o regime de criação ( 1 = a pasto, 2 = semi-confinado, 3 = confinado, 4 = com mudança no sistema ao longo da criação); $\beta$, um vetor de efeitos fixos; $Z$, uma matriz de incidência de efeitos genéticos aditivos diretos; $\alpha$, um vetor de efeitos genéticos aditivos diretos; e $\varepsilon$, um vetor de erros aleatórios associados ao vetor de observações.

\section{Resultados e Discussão}

As estimativas para os parâmetros das equações para os animais selecionados (Tabela 1) encontramse dentro de limites aceitáveis e corroboram a bibliografia consultada (Rosa et al., 1978; Ludwig et al., 1981; Nobre et al., 1987; Perotto et al., 1992; Freitas et al., 1997; Oliveira et al., 2000; Lobo \& Martins Filho, 2002).

O número de animais e de rebanhos analisados dentro da mesma raça apresentou pouca diferença entre os modelos estudados (Tabela 2), com exceção do modelo de Richards, que teve o menor número de convergências, reduzindo consideravelmente tanto o número de animais quanto o de rebanhos a serem utilizados no cálculo dos componentes de variância. Apesar de pequena, a variação entre modelos poderia afetar as estimativas dos componentes de variância, pois representariam a perda de informações importantes, não somente pela eliminação de animais, mas também pelas próprias características da amostra, com a eliminação de efeitos ambientais aos quais os animais retirados estariam sujeitos.

Esperava-se um padrão de comportamento para as estimativas dos componentes genéticos de acordo com os valores observados na revisão bibliográfica: a herdabilidade para o peso assintótico (a) seria de média a alta, por representar o peso final, que é uma característica de carcaça; a herdabilidade para o coeficiente de maturidade $(k)$, por sua vez, seria de baixa a alta, pois estaria fortemente influenciada por efeitos ambientais; a constante de integração $(b)$ apresentaria herdabilidade de média a alta no caso da curva de Brody, pois representaria o peso ao nascimento do animal, calculado por $(a-b)$ (Brown et al., 1976; DeNise \& Brinks, 1985; Koenen \& Groen, 1996) para esta curva. A tendência das correlações genéticas entre $a$ e $k$ seria negativa, pois animais mais pesados tenderiam a taxas de maturação menores que animais mais leves; entre $a$ e $b$, para a curva de Brody, seria positiva, justificando que animais nascidos mais pesados tendem a maiores pesos assintóticos.

De fato, os valores de herdabilidade encontrados para o parâmetro a (Tabelas 3, 4 e 5) foram, em geral, compatíveis com os reportados na literatura, que variam de $0,21 \pm 0,07$ (Koenen \& Groen, 1996) a 0,57 $\pm 0,11$ (Brinks et al., 1964), exceto o valor muito alto para a curva Brody, na raça Guzerá (0,9603 \pm 0,7250 Tabela 3), ou muito baixo, para a mesma curva, na raça Nelore Mocho (0,0175 \pm 0,2899 - Tabela 5).

O parâmetro $b$ também apresentou herdabilidade muito variada entre as raças e curvas estudadas: de 0,9722 \pm 0,7262 (Tabela 3), para a curva Brody, na raça Guzerá, a 0,0165 \pm 0,2873 (Tabela 5), para a mesma curva, na raça Nelore Mocho. Os valores encontrados por outros autores variaram de 0,24 \pm 0,26 (DeNise \& Brinks, 2002) a 0,62 $\pm 0,34$ (Brown et al., 1976), entre diferentes curvas para raças européias. Os valores extremos encontrados neste estudo são de difícil interpretação. 
Tabela 1 - Valores médios das estimativas dos parâmetros das equações não-lineares estudadas, de acordo com a raçac

Table 1 - Parameters estimates means for studied non-linear equations according to the breed ${ }^{c}$

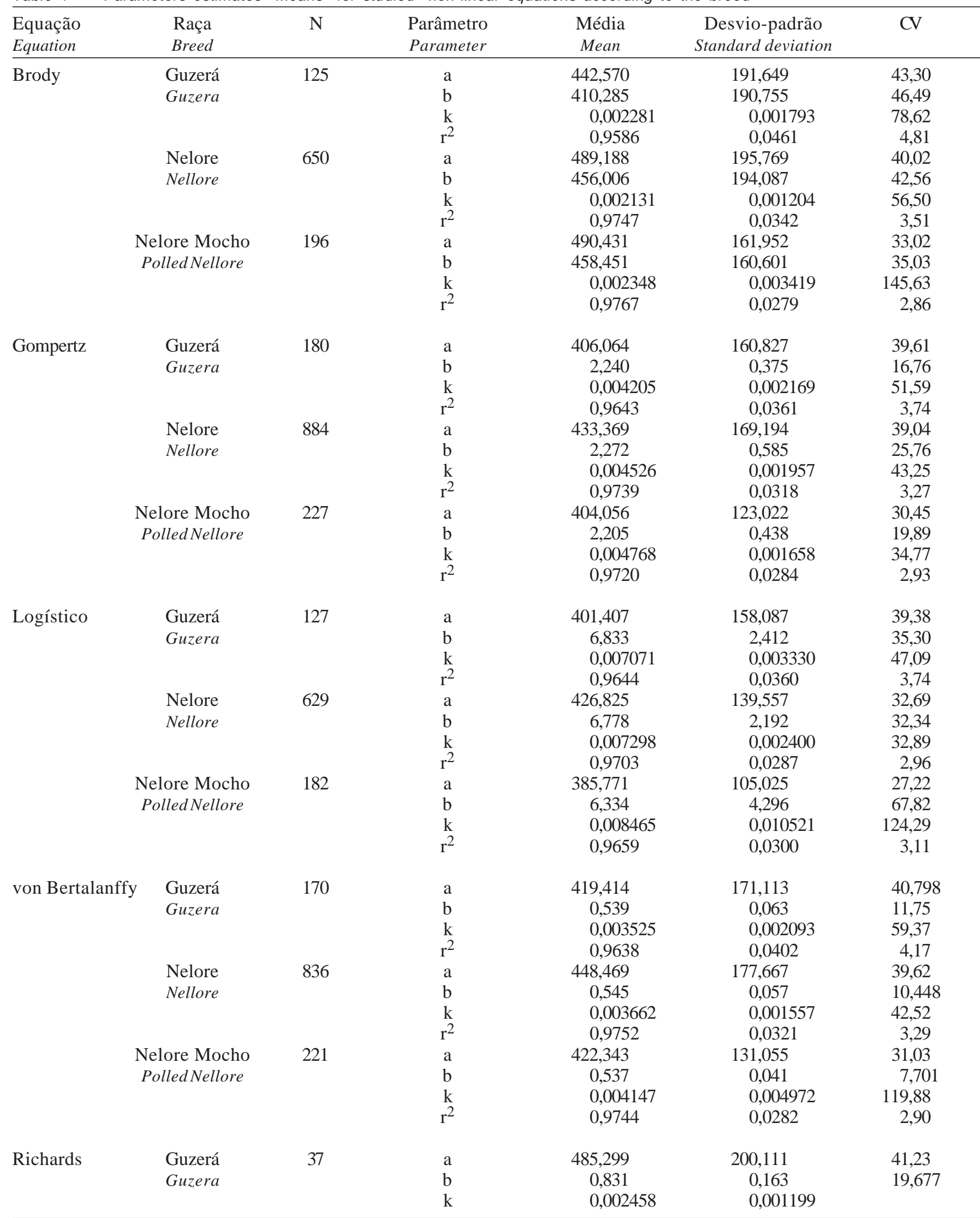


Continuação da Tabela 1 ...

\begin{tabular}{|c|c|c|c|c|c|c|}
\hline $\begin{array}{l}\text { Equação } \\
\text { Equation }\end{array}$ & $\begin{array}{l}\text { Raça } \\
\text { Breed }\end{array}$ & $\mathrm{N}$ & $\begin{array}{l}\text { Parâmetro } \\
\text { Parameter }\end{array}$ & $\begin{array}{l}\text { Média } \\
\text { Mean }\end{array}$ & $\begin{array}{l}\text { Desvio-padrão } \\
\text { Standard deviation }\end{array}$ & $\mathrm{CV}$ \\
\hline & & & $\mathrm{m}$ & 1,597329 & 1,055661 & 66,09 \\
\hline & & & $r^{2}$ & 0,9793 & 0,0363 & 3,71 \\
\hline & Nelore & 276 & $\mathrm{a}$ & 489,714 & 190,412 & 38,88 \\
\hline & Nelore & & $\mathrm{b}$ & 0,858 & 0,156 & 18,199 \\
\hline & & & $\mathrm{k}$ & 0,002313 & 0,001283 & 55,47 \\
\hline & & & $\mathrm{m}$ & 1,490664 & 1,761597 & 118,17 \\
\hline & & & $r^{2}$ & 0,9863 & 0,0185 & 1,88 \\
\hline & Nelore Mocho & 109 & $\mathrm{a}$ & 478,250 & 155,831 & 32,58 \\
\hline & Polled Nelore & & b & 0,867 & 0,148 & 17,132 \\
\hline & & & $\mathrm{k}$ & 0,002450 & 0,001367 & 55,80 \\
\hline & & & $\mathrm{m}$ & 1,474250 & 1,784523 & 121,05 \\
\hline & & & $\mathrm{r}^{2}$ & 0,9848 & 0,0165 & 1,68 \\
\hline
\end{tabular}

${ }^{\mathrm{c}} \mathrm{N}=$ número de animais analisados; $\mathrm{CV}=$ coeficiente de variação; $\mathrm{r}^{2}=$ coeficiente de ajuste.

${ }^{c} N=$ total of analyzed animals; $C V=$ coefficient of variation; $r^{2}=$ adjust coefficient.

A taxa de maturidade $(k)$ apresentou herdabilidade variável de 0,6477 \pm 7,3983, para o modelo Richards, para a raça Guzerá (Tabela 3), a 0,1970 \pm 0,1444 para a curva Logística, para a raça Nelore (Tabela 4), enquanto, na literatura, este parâmetro varia de 0,02 $\pm 0,05$ (Koenen \& Groen, 1996) a 0,33 \pm 0,25 (Brown et al., 1976).

Para o parâmetro $m$, presente apenas na curva de Richards, a herdabilidade variou de 0,9419 \pm 7,8280, para a raça Guzerá (Tabela 3), a 0,1456 \pm 0,7275, para a raça Nelore Mocho (Tabela 5), enquanto o valor encontrado na literatura foi de 0,21 $\pm 0,26$ (DeNise \& Brinks, 1985).

A correlação genética esperada entre o peso assintótico ( $a$ ) e a taxa de maturidade $(k)$ seria negativa, pois animais mais pesados tenderiam a ter taxa de maturação menor que animais mais leves, que, por sua vez, atingiriam a maturidade em idades menores. Essa tendência foi observada para a maioria das curvas nas raças estudadas (Tabelas 3, 4 e 5). Entretanto, não foi observada para a curva Richards, na raça Guzerá (Tabela 3), e para as curvas Logística e Von Bertalanffy, na raça Nelore Mocho (Tabela 5), cujas correlações foram positivas, com um comportamento de difícil explicação, possivelmente em razão de problemas de amostragem.

Uma vez que a constante de integração $(b)$ poderia ser interpretada como o peso ao nascimento para a curva de Brody (DeNise \& Brinks, 1985), estimado por $(a-b)$, esperava-se que essa variabilidade tivesse correlação genética positiva alta com o parâmetro $a$, que representa o peso assintótico, pois animais nascidos mais pesados tenderiam a ter maior peso adulto que aqueles nascidos mais leves. De fato, todos os valores apresentaram este comportamento (Tabelas 2, 3 e 4), como reportado por Koenen \& Groen (1996), que relataram correlação genética de 0,19 $\pm 0,20$, divergindo dos dados obtidos por DeNise \& Brinks (1985), que encontraram $-0,84 \pm 0,52$. A interpretação do peso ao nascimento a partir de $(a-b)$ não poderia ser feita para a curva de Richards (DeNise \& Brinks, 1985).

A correlação genética entre a constante de integração $(b)$ e a taxa de maturidade ( $k$ ) oscilou tanto em valor quanto em sentido, variando de -0,9950, para a curva de Richards, na raça Nelore (Tabela 4), até 0,8363, para a curva Von Bertalanffy, na raça Nelore Mocho (Tabela 5). A mesma tendência variável foi encontrada na literatura, com valores de - 0,01 $\pm 0,57$ (Koenen \& Groen, 1996), 0,82 \pm 1,05 e - 0,97 $\pm 1,78$ (DeNise \& Brinks, 1985), demonstrando que não haveria uma relação genética direta facilmente interpretável entre estes parâmetros.

As relações entre os parâmetros da curva de Richards foram de difícil interpretação, pois algumas mudaram de sentido e de intensidade, problema que também foi relatado por DeNise \& Brinks (1985), que destacaram que a inclusão do parâmetro de inflexão variável $(m)$ faria com que os parâmetros $b$ e $k$ perdessem sua interpretação biológica simples.

As altas herdabilidades e os altos desvios-padrão para suas estimativas (Tabelas 3, 4 e 5) foram causados pelas características das amostras estudadas (Tabela 2).

Os pais e as mães dos animais analisados não possuíam registro de produção. O coeficiente de 


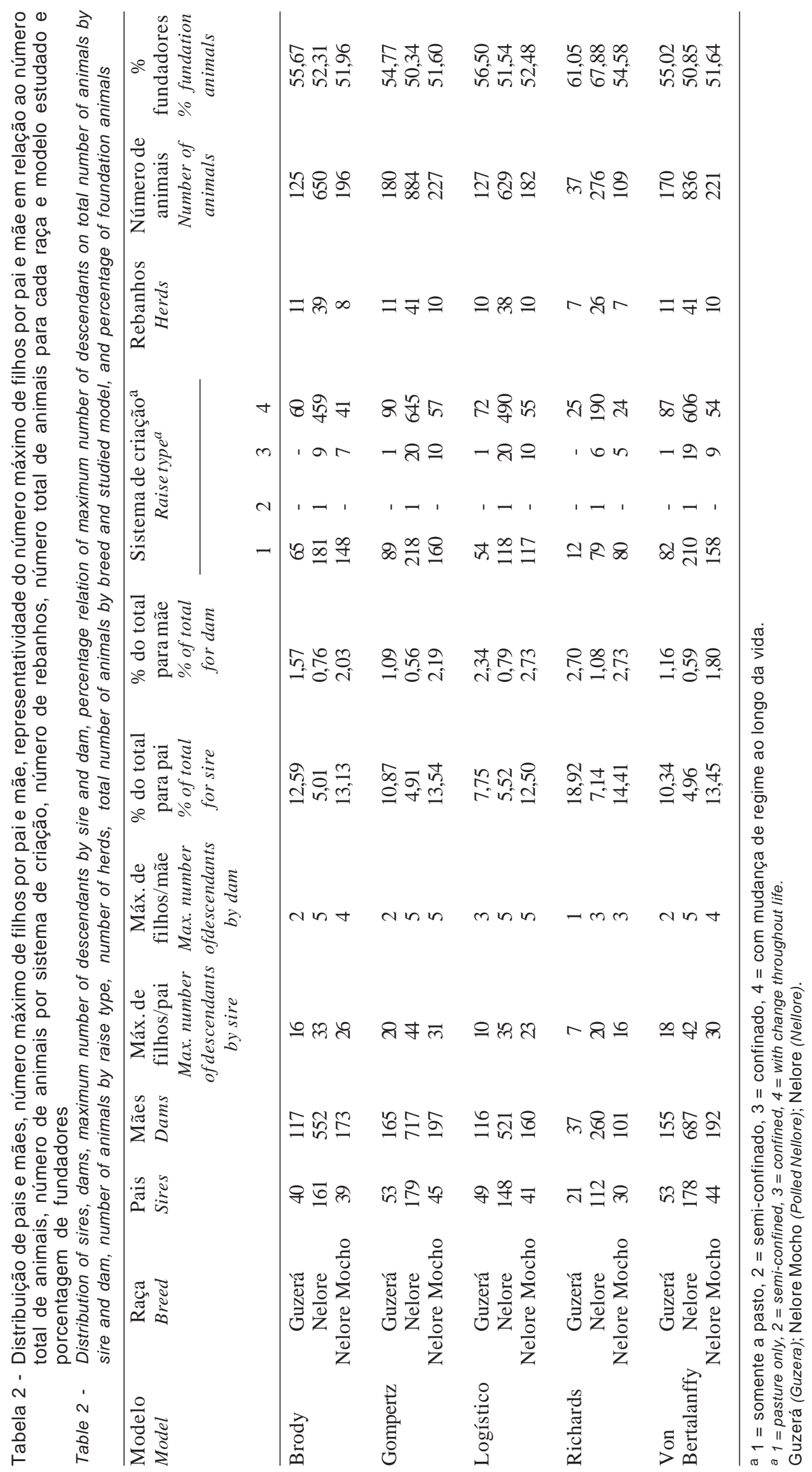

R. Bras. Zootec., v.34, n.6, p.2280-2289, 2005 (supl.) 
Tabela 3 - Herdabilidade (diagonal principal) e correlações fenotípica (diagonal superior) e genética (diagonal inferior) de bovinos da raça Guzera, para os parâmetros dos modelos não-lineares

Table 3 - Heritability (principal diagonal), phenotypic (superior diagonal), and genetic correlations (inferior diagonal) of nonlinear models parameters for Guzerá breed

\begin{tabular}{|c|c|c|c|c|c|}
\hline \multirow[b]{2}{*}{$\begin{array}{l}\text { Modelo } \\
\text { Model }\end{array}$} & \multirow[b]{2}{*}{$\begin{array}{l}\text { Parâmetro } \\
\text { Parameter }\end{array}$} & \multicolumn{4}{|c|}{$\begin{array}{l}\text { Parâmetro } \\
\text { Parameter }\end{array}$} \\
\hline & & a & $\mathrm{b}$ & k & $\mathrm{m}$ \\
\hline Brody & $\begin{array}{l}\mathrm{a} \\
\mathrm{b} \\
\mathrm{k}\end{array}$ & $\begin{array}{c}0,9603( \pm 0,7250) \\
1,0000 \\
-0,9496\end{array}$ & $\begin{array}{c}0,9992 \\
0,9722( \pm 0,7262) \\
-0,9496\end{array}$ & $\begin{array}{c}-0,6422 \\
-0,6363 \\
0,4044( \pm 0,6438)\end{array}$ & \\
\hline Gompertz & $\begin{array}{l}\mathrm{a} \\
\mathrm{b} \\
\mathrm{k}\end{array}$ & $\begin{array}{c}0,4780( \pm 0,3640) \\
0,7562 \\
-0,4127\end{array}$ & $\begin{array}{c}0,6863 \\
0,4176( \pm 0,3786) \\
-0,3075\end{array}$ & $\begin{array}{c}-0,5128 \\
-0,1753 \\
0,2136( \pm 0,2207)\end{array}$ & \\
\hline Logístico & $\begin{array}{l}\mathrm{a} \\
\mathrm{b} \\
\mathrm{k}\end{array}$ & $\begin{array}{c}0,1248( \pm 0,6162) \\
-0,9381 \\
-0,0763\end{array}$ & $\begin{array}{c}0,7062 \\
0,0707( \pm 0,5826) \\
0,1340\end{array}$ & $\begin{array}{c}-0,5496 \\
-0,1846 \\
0,2894( \pm 0,5640)\end{array}$ & \\
\hline von Bertalanffy & $\begin{array}{l}\mathrm{a} \\
\mathrm{b} \\
\mathrm{k}\end{array}$ & $\begin{array}{c}0,2571( \pm 0,3883) \\
0,8470 \\
-0,2032\end{array}$ & $\begin{array}{c}0,6634 \\
0,2168( \pm 0,3788) \\
-0,1281\end{array}$ & $\begin{array}{c}-0,5130 \\
-0,2696 \\
0,1970( \pm 0,3070)\end{array}$ & \\
\hline Richards & $\begin{array}{l}\mathrm{a} \\
\mathrm{b} \\
\mathrm{k} \\
\mathrm{m}\end{array}$ & $\begin{array}{c}0,7267( \pm 7,0217) \\
-0,7883 \\
0,0494 \\
0,8577\end{array}$ & $\begin{array}{c}-0,6964 \\
0,9230( \pm 8,7838) \\
-0,1769 \\
-0,9917\end{array}$ & $\begin{array}{c}-0,1349 \\
-0,1882 \\
0,6477( \pm 7,3983) \\
0,1515\end{array}$ & $\begin{array}{c}0,8170 \\
-0,9766 \\
0,0822 \\
0,9419( \pm 7,8280)\end{array}$ \\
\hline
\end{tabular}

Tabela 4 - Herdabilidade (diagonal principal) e correlações fenotípica (diagonal superior) e genética (diagonal inferior) de bovinos da raça Nelore, para os parâmetros dos modelos não-lineares

Table 4 - Heritability (principal diagonal), phenotypic (superior diagonal), and genetic correlations (inferior diagonal) of nonlinear models parameters for Nelore breed

\begin{tabular}{|c|c|c|c|c|c|}
\hline \multirow[b]{2}{*}{$\begin{array}{l}\text { Modelo } \\
\text { Model }\end{array}$} & \multirow[b]{2}{*}{$\begin{array}{l}\text { Parâmetro } \\
\text { Parameter }\end{array}$} & \multicolumn{4}{|c|}{$\begin{array}{l}\text { Parâmetro } \\
\text { Parameter }\end{array}$} \\
\hline & & $\mathrm{a}$ & $\mathrm{b}$ & k & $\mathrm{m}$ \\
\hline Brody & $\begin{array}{l}\mathrm{a} \\
\mathrm{b} \\
\mathrm{k}\end{array}$ & $\begin{array}{c}0,1044( \pm 0,1084) \\
0,9999 \\
-0,9347\end{array}$ & $\begin{array}{c}0,9989 \\
0,0926( \pm 0,1074) \\
-0,9385\end{array}$ & $\begin{array}{c}-0,7007 \\
-0,6909 \\
0,2204( \pm 0,1312)\end{array}$ & \\
\hline Gompertz & $\begin{array}{l}\mathrm{a} \\
\mathrm{b} \\
\mathrm{k}\end{array}$ & $\begin{array}{c}0,1728( \pm 0,0900) \\
0,6309 \\
-0,0896\end{array}$ & $\begin{array}{c}0,2712 \\
0,0807( \pm 0,0767) \\
0,0979\end{array}$ & $\begin{array}{c}-0,4502 \\
0,3296 \\
0,2440( \pm 0,0578)\end{array}$ & \\
\hline Logístico & $\begin{array}{l}\mathrm{a} \\
\mathrm{b} \\
\mathrm{k}\end{array}$ & $\begin{array}{c}0,2117( \pm 0,1318) \\
0,6047 \\
-0,6483\end{array}$ & $\begin{array}{c}0,5297 \\
0,2162( \pm 0,1195) \\
-0,6671\end{array}$ & $\begin{array}{c}-0,4465 \\
0,0562 \\
0,1977( \pm 0,1494)\end{array}$ & \\
\hline von Bertalanffy & $\begin{array}{l}\mathrm{a} \\
\mathrm{b} \\
\mathrm{k}\end{array}$ & $\begin{array}{c}0,2084( \pm 0,1057) \\
0,7971 \\
-0,2349\end{array}$ & $\begin{array}{c}0,6197 \\
0,1107( \pm 0,0949) \\
0,0376\end{array}$ & $\begin{array}{c}-0,4955 \\
-0,1874 \\
0,4156( \pm 0,0684)\end{array}$ & \\
\hline Richards & $\begin{array}{l}\mathrm{a} \\
\mathrm{b} \\
\mathrm{k} \\
\mathrm{m}\end{array}$ & $\begin{array}{c}0,5351( \pm 0,3345) \\
-0,9947 \\
-0,0665 \\
0,9993\end{array}$ & $\begin{array}{c}-0,0820 \\
0,3751( \pm 0,2668) \\
0,0541 \\
-0,9950\end{array}$ & $\begin{array}{c}-0,3435 \\
-0,5301 \\
0,2585( \pm 0,2933) \\
-0,0651\end{array}$ & $\begin{array}{c}0,2383 \\
-0,8202 \\
0,2446 \\
0,6996( \pm 0,3287)\end{array}$ \\
\hline
\end{tabular}


Tabela 5 - Herdabilidade (diagonal principal) e correlações fenotípica (diagonal superior) e genética (diagonal inferior) de bovinos da raça Nelore Mocho, para os parâmetros dos modelos não-lineares

Table 5 - Heritability (principal diagonal), phenotypic (superior diagonal), and genetic correlations (inferior diagonal) of nonlinear models parameters for Nelore Mocho breed

\begin{tabular}{|c|c|c|c|c|c|}
\hline \multirow[b]{2}{*}{$\begin{array}{l}\text { Modelo } \\
\text { Model }\end{array}$} & \multirow[b]{2}{*}{$\begin{array}{l}\text { Parâmetro } \\
\text { Parameter }\end{array}$} & \multicolumn{4}{|c|}{$\begin{array}{l}\text { Parâmetro } \\
\text { Parameter }\end{array}$} \\
\hline & & $\mathrm{a}$ & $\mathrm{b}$ & k & $\mathrm{m}$ \\
\hline Brody & $\begin{array}{l}\mathrm{a} \\
\mathrm{b} \\
\mathrm{k}\end{array}$ & $\begin{array}{c}0,0175( \pm 0,2899) \\
1,0000 \\
-0,0831\end{array}$ & $\begin{array}{c}0,9983 \\
0,0165( \pm 0,2873) \\
-0,0832\end{array}$ & $\begin{array}{c}-0,1691 \\
-0,1660 \\
0,3419( \pm 0,3386)\end{array}$ & \\
\hline Gompertz & $\begin{array}{l}\mathrm{a} \\
\mathrm{b} \\
\mathrm{k}\end{array}$ & $\begin{array}{c}0,4934( \pm 0,3541) \\
0,3773 \\
-0,3894\end{array}$ & $\begin{array}{c}0,4370 \\
0,9356( \pm 0,3850) \\
0,3027\end{array}$ & $\begin{array}{c}-0,4803 \\
0,1137 \\
0,5331( \pm 0,2924)\end{array}$ & \\
\hline Logístico & $\begin{array}{l}\mathrm{a} \\
\mathrm{b} \\
\mathrm{k}\end{array}$ & $\begin{array}{c}0,3658( \pm 0,4490) \\
0,2517 \\
0,6256\end{array}$ & $\begin{array}{c}0,4007 \\
0,8962( \pm 0,6336) \\
0,7660\end{array}$ & $\begin{array}{c}0,0790 \\
0,4432 \\
0,5167( \pm 0,4747)\end{array}$ & \\
\hline Von Bertalanffy & $\begin{array}{l}\mathrm{a} \\
\mathrm{b} \\
\mathrm{k}\end{array}$ & $\begin{array}{c}0,7431( \pm 0,3604) \\
0,5169 \\
0,1535\end{array}$ & $\begin{array}{c}0,4942 \\
0,4033( \pm 0,2859) \\
0,8363\end{array}$ & $\begin{array}{c}-0,0508 \\
0,0913 \\
0,2480( \pm 0,2326)\end{array}$ & \\
\hline Richards & $\begin{array}{l}\mathrm{a} \\
\mathrm{b} \\
\mathrm{k} \\
\mathrm{m}\end{array}$ & $\begin{array}{c}0,4202( \pm 0,5838) \\
0,7529 \\
-0,3539 \\
-0,4274\end{array}$ & $\begin{array}{c}0,3493 \\
0,5820( \pm 0,7109) \\
-0,5422 \\
-0,9167\end{array}$ & $\begin{array}{c}-0,4274 \\
-0,6326 \\
0,5971( \pm 0,3944) \\
0,5301\end{array}$ & $\begin{array}{c}-0,1388 \\
-0,8042 \\
0,5803 \\
0,1456( \pm 0,7275)\end{array}$ \\
\hline
\end{tabular}

consangüinidade médio para as amostras estudadas foi zero e a moda para o número de descendentes foi um. Os reprodutores, por sua vez, apresentaram conexão genética ou apresentaram em número muito pequeno. Os animais que não possuíam conexão genética foram considerados animais fundadores do rebanho e aqueles que possuíam registro de produção estavam muito espaçados ao longo do tempo estudado, o que diminuiu o número de conexões para os efeitos ambientais. Para os diferentes modelos nãolineares, dentro de uma mesma raça, foram dispostas diversas amostras para estimação dos componentes de variância, com diferentes conteúdos em efeitos fixos e aleatórios e estrutura de relacionamento genético. Considerando estas características, a estrutura das amostras estudadas (Tabela 2) contribuiu significativamente para os elevados desvios-padrão das estimativas dos componentes genéticos.

Reverter \& Kaiser (1997) demonstraram que a influência da proporção de indivíduos fundadores sobre o erro-padrão da herdabilidade não seria linear, como acreditava-se, mas sim quadrático, de modo que o erro padrão diminuiria com uma proporção de fun- dadores de 5 a 30\%, atingindo mínimo de 20 a 40\%, e aumentaria com proporções acima de 40\%. Esses autores encontraram também correlação significativa entre a taxa de reposição dos machos e a proporção macho-fêmea e notaram que maiores desvios-padrão estariam associadas a maiores proporções, de forma que, na relação 1:20, foram encontrados maiores desvios-padrão que na relação 1:10. Outros motivos seriam a baixa razão entre sexos, o pequeno número de descendentes por fundador, a ausênica de informação de produção para os fundadores e o baixo número de descendentes por fundador (Mallinckrodt et al., 1997; Reverter \& Kaiser, 1997; Tosh \& Wilton, 1994).

Além do tamanho da população e de sua estrutura de relacionamento, Garrick et al. (1989) salientaram que seria difícil calcular os erros-padrão para as estimativas de parâmetros genéticos pelo fato de a distribuição de sua amostragem não ser conhecida. Além disso, intervalos de confiança para estimativas de herdabilidade seriam raramente relatados ou necessitariam de métodos apropriados para serem estimados ou calculados, como o método "Bootstrap”, 
empregado por Mallinckrodt et al. (1997). De acordo com Tosh \& Wilton (1994), a obtenção das variâncias dos erros de predição diretamente da inversa da matriz de coeficiente seria impraticável, mas seria possível por outros métodos ou por informações auxiliares.

Com essas considerações, seria difícil encontrar o máximo global da função de verossimilhança em uma superfície dimensional plana, com pouca declividade ou com vários máximos locais (Verneque, 1994) pelo método livre de derivada, proposto por Smith \& Graser (1986) e Graser et al. (1987), empregado pelo programa utilizado, o que aumentaria o erro de predição e diminuiria a taxa de convergência do algoritmo.

$O$ reduzido número de trabalhos sobre a herdabilidade dos parâmetros de curvas de crescimento de modelos não-lineares impossibilitou uma análise comparativa mais aprofundada dos resultados.

O uso das informações obtidas nos rebanhos estudados em um programa de melhoramento genético visando a alteração da curva de crescimento dos animais deve ser feito com grande cautela, uma vez que as características a serem trabalhadas na modificação do formato da curva de crescimento (peso ao nascimento e taxa de maturação) seriam negativamente correlacionadas (Fitzhugh Jr., 1976). Neste caso, os índices de seleção restritos poderiam ser utilizados com mais sucesso (Rosa et al., 1979; Atchley et al., 1997; Dzama et al., 2001). Brown et al. (1976) destacaram que a taxa de maturidade $(k)$ poderia ser o único parâmetro disponível para o trabalho da indústria.

Os mesmos modelos apresentaram diferentes estimativas de herdabilidade entre as raças estudadas e, dentro da mesma raça, diferentes modelos também tiveram esta variabilidade, sugerindo que deve-se ter muita cautela na escolha da curva em um programa de melhoramento genético, pois animais que não apresentem convergência por um modelo e que, portanto, possam ser eliminados das análises, poderiam apresentar convergência em outro modelo e representar importante perda genética em um programa de melhoramento genético. Além disso, a perda de informação com a eliminação de determinado animal, observando-se os efeitos genéticos e ambientais, poderia resultar em um viés na estimação da importância destes efeitos.

\section{Conclusões}

As raças Guzerá e Nelore Mocho apresentaram grande variabilidade para os valores de variância e covariância genéticas para os parâmetros dos modelos das curvas de crescimento utilizadas, prejudicando a análise e a correta interpretação do comportamento desses componentes na população. Esta variabilidade resultou, principalmente, das limitações das amostras estudadas para essas raças.

No caso da raça Nelore, os resultados foram consistentes, demonstrando a possibilidade de aproveitamento das informações obtidas para uso em programas de melhoramento genético e do julgamento do mérito genético dos animais.

\section{Literatura Citada}

ATCHLEY,W.T.; XU, S.; COWLEY, D.E. Altering developmental trajectories in mice by restricted index selection. Genetics, v.146, p.629-640, 1997.

von BERTALANFFY, L. Quantitative laws in metabolism and growth. Quarterly Review of Biology, v.32, p.218, 1957.

BRINKS, J.S.; CLARK, R.T.; KEIFER, N.M. et al. Estimates of genetic, environmental and phenotypic parameters in range Hereford females. Journal of Animal Science, v.23, p.711-723, 1964.

BRODY, S. Bioenergetics and growth. New York: Rheinhold Pub. Corp., 1945. 645p.

BROWN, J.E.; FITZHUGH Jr., H.A.; CARTWRIGHT, T.C. A comparison of nonlinear models for describing weight-age relationships in cattle. Journal of Animal Science, v.42, n.4, p.810-818, 1976.

DZAMA, K.; WALTER, J.P.; RUVUNA, F. et al. Index selection of beef cattle for growth and milk production using computer simulation modelling. South African Journal of Animal Science, v.31, n.2, p.65-75, 2001

DENISE, R.S.K.; BRINKS, J.S. Genetic and environmental aspects os growth curve parameters in beef cows. Journal of Animal Science, v.61, n.6, p.1431-1440, 1985.

FITZHUGH JR., H.A. Analysis of growth curves and strategies for altering their shape. Journal of Animal Science, v.42, n.4, p.1036-1051, 1976.

FREITAS, A.F.; DURÃES, M.C.; TEIXEIRA, N.M. Curvas de crescimento de novilhas da raça Holandesa mantidas em regime de confinamento. Arquivos Brasileiros de Medicina Veterinária e Zootecnia, v.49, n.1, p.85-93, 1997.

GARRICK, D.J.; POLLAK, E.J.; QUAAS, R.L. et al. Variance heterogeneity in direct and maternal weight traits by sex and percent purebred for Simmental-sired calves. Journal of Animal Science, v.67, p.2515, 1989.

GOONEWARDENE, L.A.; BERG, R.T.; HARDIN, R.T. A growth study of beef cattle. Canadian Journal of Animal Science, v.61, p.1041-1048, 1981.

GRASER, H.U.; SMITH, S.P., TIER, B.A. Derivative-free approach for estimating variance components in animal models 
by restricted maximum likelihood. Journal of Animal Science, v.64, p.1362-1370, 1987.

HOFFMAN, R.; VIEIRA, S. Uma introdução à econometria. 3.ed. São Paulo: HUCITEC, 1998. 379p.

KAPS, M.; HERRING, W.O.; LAMBERSON, W.R. Genetic and environmental parameters for traits derived from the Brody growth curve and their relationships with weaning weight in Angus cattle. Journal of Animal Science, v.78, p.1436-1442, 2000.

KOENEN, E.P.C.; GROEN, A.F. Genetic analysis of growth patterns of black and white dairy heifers. Journal of Dairy Science, v.79, p.495-501, 1996.

KROLL, L.B.; TORNERO, M.T.T. Modelo de Gompertz com sazonalidade e autocorrelação nos erros para ajuste do crescimento ponderal em vaca leiteira. Pesquisa Agropecuária Brasileira, v.29, n.5, p.707-711, 1994.

KVÅLSETH, T.O. Cautionary note about $\mathrm{R}^{2}$. The Amerian Statistician, parte 1, v.39, n.4, p.279-285, 1985.

LÔBO, R.N.B.; MARTINS FILHO, R. Avaliação de curvas de crescimento de bovinos da raça Nelore. In: REUNIÃO ANUAL DA SOCIEDADE BRASILEIRA DE ZOOTECNIA, 39., 2002, Recife. Anais... Recife: Sociedade Brasileira de Zootecnia, 2002. CD-ROM.

LUDWIG, A.; SILVA, M.A.; OLIVEIRA, L.M. Ajustamento de modelos estatísticos exponenciais ao crescimento de gado nelore. Pesquisa Agropecuária Brasileira, v.16, n.2, p.297-302, 1981.

MALLINCKRODT, C.H.; GOLDEN, B.L.; REVERTER, A. Approximate confidence intervals for heritability from method R estimates. Journal of Animal Science, v.75, p.20412046, 1997.

MAZZINI, A.R.A. Análise da curva de crescimento de machos Hereford considerando heterogeneidade de variâncias e autocorrelação dos erros. Lavras: Universidade Federal de Lavras, 2001. 94p. Dissertação (Mestrado em Experimentação Agrícola) - Universidade Federal de Lavras, 2001.

MEYER, K. DFREML - Version 3.0 b - user notes. < http:// agbu.une.edu.au/ kmeyer//dfreml.html > Acessado em 13.07.2000. 29p.

NELDER, J.A. The fitting of a generalization of the logistic curve. Biometrics, v.17, p.89-94, 1961.

NOBRE, P.R.C.; ROSA, A.N.; SILVA, L.O.C. et al. Curvas de crescimento de gado nelore ajustadas para diferentes frequências de pesagens. Pesquisa Agropecuária Brasileira, v.22, n.9/10, p.1027-1037, 1987.

OLIVEIRA, H.N.; LÔBO, R.B.; PEREIRA, C.S. Comparação de modelos não-lineares para descrever o crescimento de fêmeas da raça Guzerá. Pesquisa Agropecuária Brasileira, v.35, n.9, p.1843-1851, 2000.

OWENS, F.N.; DUBESKI, P.; HANSON, C.F. Factors that alter the growth and development of ruminants. Journal of Animal Science, v.71, p.3138-3150, 1993.
PEROTTO, D.; CUE, R.I.; LEE, A.J. Comparison of nonlinear functions for describing the growth curve of three genotypes of dairy catle. Canadian Journal of Animal Science, v.72, p.773-782, 1992.

PEROTTO, D.; CUE, R.I.; LEE, A.J. et al. Additive and nonadditive genetic effects of growth-curve parameters of Holstein, Ayrshire and crossbreed females. Canadian Journal of Animal Science, v.74, p.401-409, 1994.

PEROTTO, D.; CASTANHO, M.J.P.; CUBAS, A.C. et al. Efeitos genéticos sobre as estimativas dos parâmetros das curvas de crescimento de fêmeas bovinas Gir, Holandês x Gir e Holandês x Guzerá. Revista Brasileira de Zootecnia, v. 26, n. 4, p.719-725, 1997.

REVERTER, A.; KAISER, C.J. The role of different pedigree structures on the sampling variance of heritability estimates. Journal of Animal Science, v.75, p.2355-2361, 1997.

RICHARDS, F.J. A flexible growth function for empirical use. Journal of Experimental Botany, v.10, n.29, p.290-300, 1959.

ROSA, A.N.; SILVA, M.A.; LUDWIG, A. Parâmetros genéticos e fenotípicos de pesos corporais ajustados pela curva de crescimento de animais da raça Nelore. Revista Brasileira de Zootecnia, v.7, n.2, p. 329-345, 1978.

ROSA, A.N.; SILVA, M.A.; MILAGRES, J.C. et al. Mudança da curva de crescimento de animais da raça Nelore mediante o uso de índices de seleção. Revista Brasileira de Zootecnia, v. 8, n.4, p.610-621, 1979.

STATISTICAL ANALYSES SYSTEM - SAS. SAS/STAT User's guide. Version 8.2. 4.ed. v.2. Cary: 2000.

SMITH, S.P.; GRASER, H.U. Estimation of variance components in a class of mixed models by restricted maximum likelihood. Journal of Dairy Science, v.64, n.4, p.1156-1165, 1986.

TOSH, J.J.; WILTON, J.W. Effects of data structure on variance of prediction error and accuracy of genetic evaluation. Journal of Animal Science, v.72, p.2568-2577, 1994.

VERNEQUE, R.S. Procedimentos numéricos e estimação de componentes de covariância em análise multivariada pelo método da máxima verossimilhança restrita - Modelos mistos aplicados ao melhoramento animal. Piracicaba: Escola Superior de Agricultura “Luiz de Queiroz”, 1994. 157p. Tese (Doutorado em Estatística e Experimentação Agronômica) - Escola Superior de Agricultura "Luiz de Queiroz”, 1994.

WINSOR, C.P. The Gompertz curve as a growth curve. Proceedings of the National Academy of Science, v.18, p.1-17, 1932.

Recebido em: 13/04/04 Aceito em: 15/07/05 